\title{
Verwendung von Hydrogelen für die Detektion von Wasserstoffperoxid in der Gasphase und in Aerosolen
}

\author{
A. Müller ${ }^{1}$ S. Klötzer ${ }^{2}$, M. Decker ${ }^{1}$, F.-M. Matysik ${ }^{3}$, W. Vonau ${ }^{1}$ \\ ${ }^{1}$ Kurt-Schwabe-Institut für Mess- und Sensortechnik e.V. Meinsberg, Waldheim, Deutschland \\ ${ }^{2}$ Hochschule Mittweida - University of Applied Sciences, Mittweida, Deutschland \\ ${ }^{3}$ Universität Regensburg, Regensburg, Deutschland \\ Kontakt: anne.mueller@ksi-meinsberg.de
}

\section{Einleitung}

Die sensitive und selektive Bestimmung von Wasserstoffperoxid $\left(\mathrm{H}_{2} \mathrm{O}_{2}\right)$ ist von großer Bedeutung für biologische, pharmazeutische, ökologische und viele andere Anwendungsbereiche. Insbesondere bei der Dekontamination von Oberflächen spielt $\mathrm{H}_{2} \mathrm{O}_{2}$ eine wichtige Rolle als Sterilisationsmittel. Je nach Anforderung wird eine hochkonzentrierte $\mathrm{H}_{2} \mathrm{O}_{2}$-Lösung entweder durch einen Verdampfer geleitet und dann mit Hilfe eines Trägergases in den zu sterilisierenden Raum eingebracht [1] oder mittels Druckluft vernebelt [2]. $\mathrm{H}_{2} \mathrm{O}_{2}$ ist daher entweder nur in der Gasphase vorhanden oder zusätzlich Inhaltsstoff von Aerosolpartikeln. Daher wird angestrebt, einen kostengünstigen Sensor zu entwickeln, der $\mathrm{H}_{2} \mathrm{O}_{2}$ Mengen sowohl in der Gasphase als auch in Aerosolen zuverlässig erfassen kann.

Für die Detektion von $\mathrm{H}_{2} \mathrm{O}_{2}$ sowohl in der Gasphase als auch in Aerosolpartikeln soll ein verbreitetes elektroanalytisches Verfahren Verwendung finden. Dabei wird die bekannte Bestimmung von $\mathrm{H}_{2} \mathrm{O}_{2}$ in Lösung mit dem Redoxmediator Preußisch Blau (PB) hinsichtlich der Messung des Analyten im Gasraum modifiziert. $\mathrm{PB}$ ist bekannt für seine ausgezeichnete katalytische Aktivität und Selektivität bei der Reduktion von Wasserstoffperoxid [3]. Ein für die Massenproduktion geeignetes und damit kostengünstiges Verfahren zur Herstellung ist der Siebdruck der PB-Arbeitselektrode. Basierend auf kommerziellen Elektrodenstrukturen wurden diese mit Hydrogelen, wie von Benedet et al. [4] beschrieben, bedeckt und für die Bestimmung von $\mathrm{H}_{2} \mathrm{O}_{2}$-Mengen in der Gasphase und von Aerosolen verwendet.

\section{Methoden und Materialien}

Um den Einfluss des Feuchthaltemittels Glycerin auf die Verdunstung von Wasser aus dem Agarosegel zu untersuchen, wurden je $2 \mathrm{ml}$ der Gelproben mit unterschiedlichen Glycerinanteilen in Bechergläsern mit einem Durchmesser von $4,1 \mathrm{~cm}$ bei Raumtemperatur und Raumfeuchte offen gelagert. Zu diesem Zweck wurde 0,2 g Agarose (Biozym LE, Biozym Scientific $\mathrm{GmbH}$, Deutschland) in $10 \mathrm{ml}$ einer 0,2 molaren KCl-Lösung (Carl Roth $\mathrm{GmbH}+$ Co.
KG, Deutschland) mit dem jeweiligen Anteil an Glycerin (wasserfrei, Merck KGaA, Deutschland) bei einer Temperatur von $90{ }^{\circ} \mathrm{C}$ gelöst und in Anlehnung an Benedet et al. [4] für 5 min auf $100^{\circ} \mathrm{C}$ erhitzt. Nach Erstarren des Agarosegels wurde der Verdunstungsprozess über vier Stunden mit gravimetrischen Messungen überwacht.

Die Beschichtung von kommerziellen Sensoren der Firma DropSens (DS710, Spanien) mit Agarosegel wurde durch Aufrakeln des Elektrolyten mit einer Metallrakel realisiert. Der Sensor wurde hierzu in einer auf $40{ }^{\circ} \mathrm{C}$ temperierten Halterung fixiert und mit einer $0,3 \mathrm{~mm}$ dicken Lochmaske bedeckt, siehe Abbildung 1. Anschließend wurde ein Tropfen eines auf $60{ }^{\circ} \mathrm{C}$ temperierten Agarosegels mit einem Glycerinanteil von $50 \%(\mathrm{v} / \mathrm{v})$ auf die erwärmte Sensoroberfläche gegeben. Im Anschluss wurde das Gel mit Hilfe der Metallrakel abgezogen und die Lochmaske vorsichtig entfernt. Der Sensor wurde schließlich auf Raumtemperatur abgekühlt und konnte für die nachfolgenden Messungen verwendet werden.

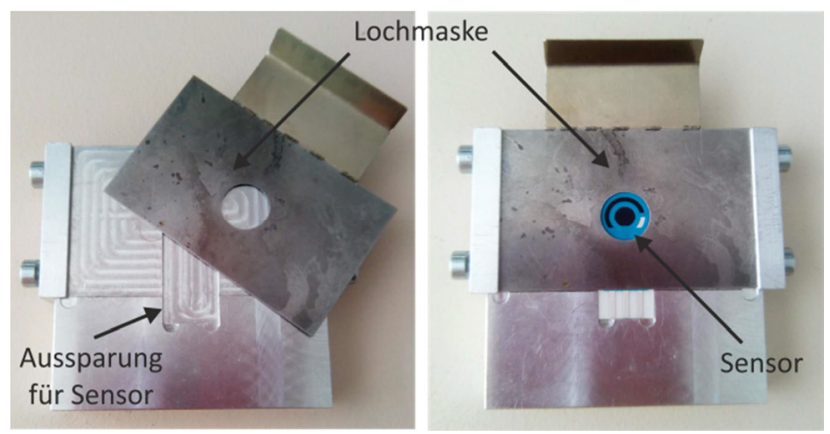

Abb. 1: Temperierbare Halterung und Lochmaske zur reproduzierbaren Gelaufbringung.

Für die Bestimmung von $\mathrm{H}_{2} \mathrm{O}_{2}$ in der Gasphase und in Aerosolen wurde eine Prüfkammer verwendet, in die eine $49,5 \%$ ige $\mathrm{H}_{2} \mathrm{O}_{2}$-Lösung (INTEROX SG-50, 49,5\%(w/w), Solvay Chemicals International SA, Belgien) mit Druckluft ( 6 bar) bei einem Durchfluss von $3 \mathrm{ml} / \mathrm{min}$ vernebelt wurde. Eine schematische Darstellung zeigt Abbildung 2. 


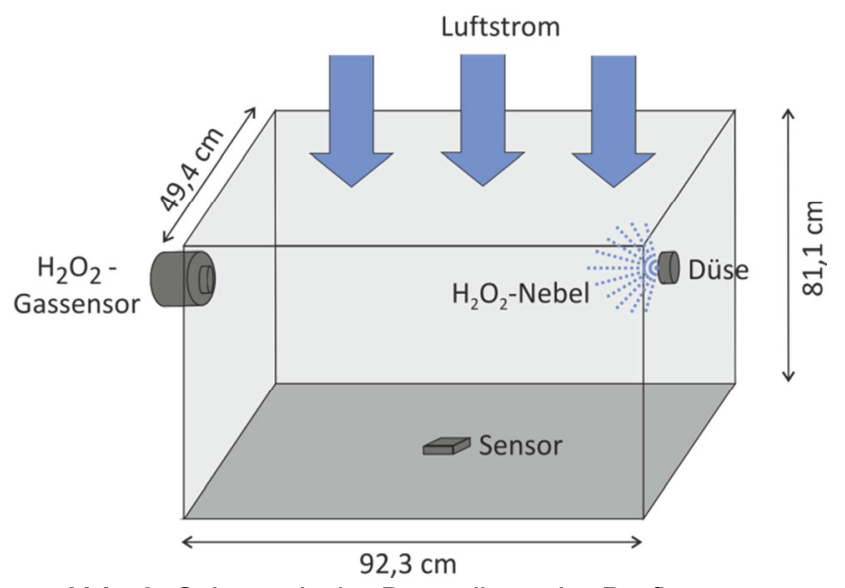

Abb. 2: Schematische Darstellung der Prüfkammer.

Die Messung erfolgte amperometrisch bei einem Potential von $-0,5 \vee$ gegenüber der ebenfalls auf der Struktur befindlichen $\mathrm{Ag} / \mathrm{AgCl}$-Referenzelektrode mit einem Potentiostaten (Interface 1000, Gamry Instruments, USA). Das angelegte Potential ist deutlich negativer als der beobachtete Reduktionspeak des PB im Bereich zwischen 0,3 V und $0 \mathrm{~V}$. Folglich wird alles vorliegende PB in seine reduzierte und katalytisch aktive Form, das preußische Weiß (PW), umgewandelt (Gleichung 1).

$\mathrm{Fe}_{4}\left[\mathrm{Fe}(\mathrm{CN})_{6}\right]_{3}+4 \mathrm{e}^{-}+4 \mathrm{~K}^{+} \rightarrow \mathrm{K}_{4} \mathrm{Fe}_{4}\left[\mathrm{Fe}(\mathrm{CN})_{6}\right]_{3}$

Die Kammer wurde zusätzlich zur Detektion von gasförmigem $\mathrm{H}_{2} \mathrm{O}_{2}$ mit einem Sensor der Firma Dräger (Dräger-Sensor $\mathrm{H}_{2} \mathrm{O}_{2} \mathrm{HC}$, Drägerwerk $A G$ \& Co. $\mathrm{KGaA}$, Deutschland) ausgestattet. Dieser Sensor misst nur gasförmiges $\mathrm{H}_{2} \mathrm{O}_{2}$ und diente als Referenz zur genauen Identifizierung des Beginns und des Endes jeder Dosierphase. Während einer Messung wurde ein permanenter Luftstrom durch die Prüfkammer geleitet, um eine konstante Atmosphäre aus gasförmigem $\mathrm{H}_{2} \mathrm{O}_{2}$ und Aerosolen zu erzeugen.

\section{Ergebnisse und Diskussion}

Die in Abbildung 3 dargestellten Ergebnisse zeigen den verdunstungsbedingten zeitlichen Gewichtsverlust der Agarosegele in Abhängigkeit vom Glycerinanteil der jeweiligen Proben. Das Ausgangsgewicht der jeweiligen Gelproben zu Beginn der Messung $\left(t_{0}\right)$ wurde hierfür auf $100 \%$ gesetzt. Aufgrund des Versuchsaufbaues kam es zu geringfügigen Schwankungen der Umgebungsbedingungen $\left(\mathrm{pH}_{2} \mathrm{O}=1000-1200 \mathrm{~Pa}\right)$, deren Einfluss auf den Ausgang der Untersuchungen jedoch vernachlässigt werden kann.

Es zeigte sich, dass niedrige Glyceringehalte bis $20 \%(\mathrm{v} / \mathrm{v})$ keinen signifikanten Einfluss auf die hohe Verdunstungsrate des Wassers im Gel besaßen. Nach $4 \mathrm{~h}$ zeigten diese Agaroseproben einen Verdunstungsverlust von etwa $12 \%$.

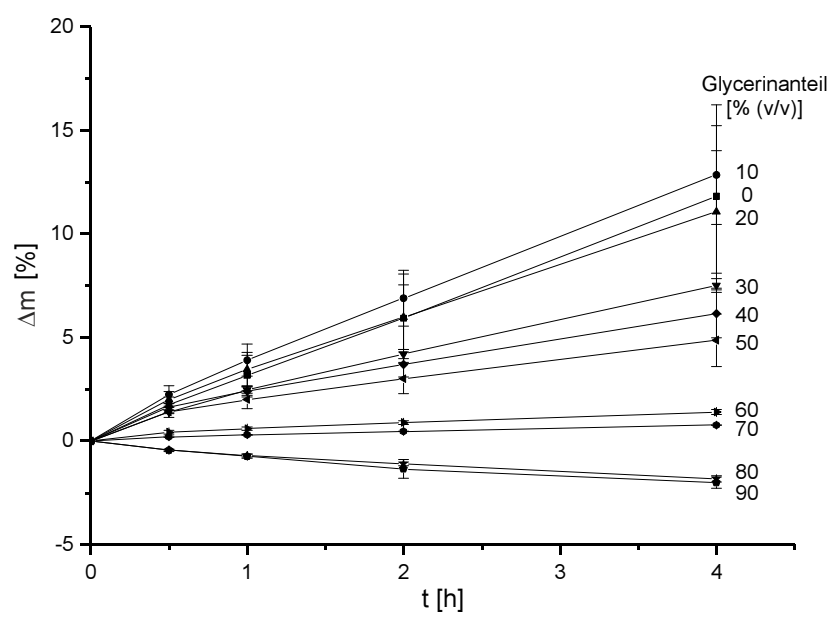

Abb. 3: Gewichtsverlust ( $\Delta \mathrm{m}[\%])$ von Agarosegelen mit unterschiedlichen Glycerinanteilen in Abhängigkeit von der Zeit, $\mathrm{n}=3$.

Mit steigendem Anteil an Feuchthaltemittel konnte die Verdunstung deutlich minimiert werden, sodass bei einem Glyceringehalt von $70 \%(\mathrm{v} / \mathrm{v})$ das Gel nach $4 \mathrm{~h}$ Lagerung nur $1 \%$ seiner Ausgangsmasse verlor.

Ab einem Glycerinanteil von $80 \%(\mathrm{v} / \mathrm{v})$ kam es in den getesteten Proben sogar zu einer Gewichtszunahme und einem Quellen des Gels aufgrund einer Wasseraufnahme aus der Umgebungsluft. Grund hierfür sind die hygroskopischen Eigenschaften von Glycerin aufgrund seiner drei hydrophilen alkoholischen Hydroxylgruppen [5].

Die Ergebnisse legen nahe, dass ein Glyceringehalt zwischen $70-80 \%(\mathrm{v} / \mathrm{v})$ gut geeignet wäre, um die Feuchtigkeit des Gels über den in der Praxis oft vorliegenden Messzeitraum von $2 \mathrm{~h}$ konstant zu halten. Jedoch konnte beobachtet werden, dass das Feuchthaltemittel nicht nur einen Einfluss auf die Verdunstung sondern auch auf die Konsistenz des Gels hatte. Mit steigendem Gehalt an Glycerin wurde das Agarosegel immer weicher bis es ab einer Konzentration von $60 \%(\mathrm{v} / \mathrm{v})$ langsam fließfähig wurde und für die geplante Anwendung nicht mehr geeignet war. Einen Kompromiss aus ausreichendem Feuchthaltevermögen und Festigkeit bot das Agarosegel mit $50 \%$ (v/v) Glycerin. Hierbei handelte es sich noch um ein festes Gel und wies nach $4 \mathrm{~h}$ noch $95 \%$ seiner Ausgangsmasse auf.

Das Agarosegel konnte mit Hilfe der Lochmaske reproduzierbar in einer Schichtdicke von 0,3 $\mathrm{mm}$ auf die planaren Sensorstrukturen aufgebracht werden. Die anschließende amperometrische Messung der Sensoren in der Prüfkammer unter Beaufschlagung mit gasförmigem $\mathrm{H}_{2} \mathrm{O}_{2}$ und Aerosolen ist in Abbildung 4 dargestellt. 


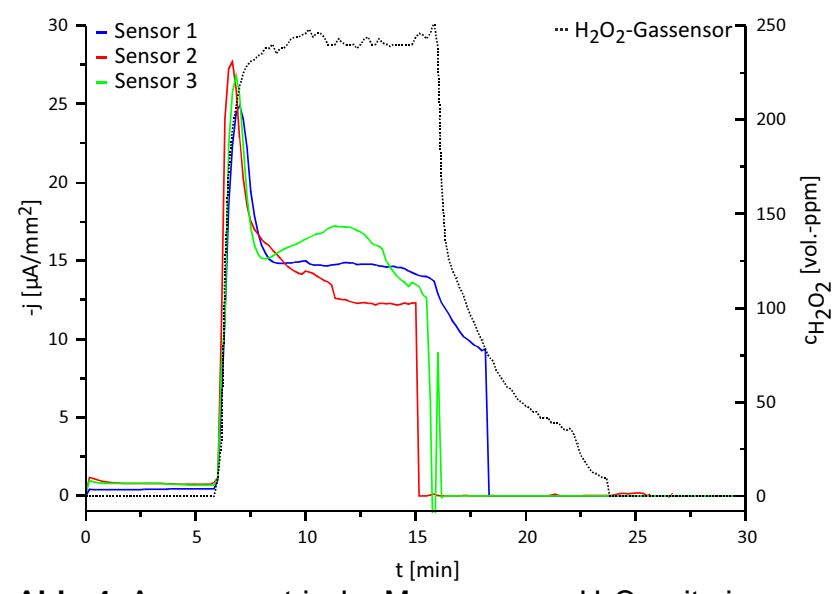

Abb. 4: Amperometrische Messung von $\mathrm{H}_{2} \mathrm{O}_{2}$ mit einem mit Agarose-Gel (50 \% (v/v) Glycerin) beschichteten Sensor bei einer Polarisationsspannung von $-0,5 \mathrm{~V}$.

Die Stromdichte der mit Agarose beschichteten Sensoren wurde während der Dosierung der gasförmigen $\mathrm{H}_{2} \mathrm{O}_{2}$-Phase und der Aerosolpartikel $\left(49,5 \% \quad \mathrm{H}_{2} \mathrm{O}_{2}\right)$ aufgezeichnet. Die Einleitung des gasförmigen $\mathrm{H}_{2} \mathrm{O}_{2}$ wurde parallel durch einen kommerziellen $\mathrm{H}_{2} \mathrm{O}_{2}$-Gassensor (gestrichelte Linie) kontrolliert und diente als Überwachungsinstrument für Beginn und Ende der Dosierphase.

Der kommerzielle Gassensor belegte, dass eine konstante Konzentration von gasförmigem $\mathrm{H}_{2} \mathrm{O}_{2}$ von ca. 240 Vol.-ppm während der Dosierphase beibehalten wurde. Im Gegensatz dazu zeigten die mit Agarose bedeckten Siebdrucksensoren zunächst einen schnellen Signalanstieg nach Beginn der $\mathrm{H}_{2} \mathrm{O}_{2}$-Einbringung. Dies deutet auf eine schnelle Reaktion von PW mit $\mathrm{H}_{2} \mathrm{O}_{2}$ auf der Elektrodenoberfläche hin (Gleichung 2).

$\mathrm{K}_{4} \mathrm{Fe}_{4}\left[\mathrm{Fe}(\mathrm{CN})_{6}\right]_{3}+2 \mathrm{H}_{2} \mathrm{O}_{2} \rightarrow \mathrm{Fe}_{4}\left[\mathrm{Fe}(\mathrm{CN})_{6}\right]_{3}+4 \mathrm{~K}^{+}+4 \mathrm{OH}^{-}$

Nach den ersten Minuten nahm das Sensorsignal ab und bildete ein Plateau. Dieses Plateau legte nahe, dass der Detektionsprozess entweder durch die Kinetik des Elektronentransfers für die elektrochemische Regeneration des PW, durch strukturelle Transformationen der PB-Pastenschicht oder durch Veränderungen innerhalb des Elektrolyten während des Messprozesses beeinträchtigt wurde. Die experimentellen Befunde zeigten, dass nicht alles ankommende $\mathrm{H}_{2} \mathrm{O}_{2}$ mit PW an der Elektrode reagiert. Eine beträchtliche Menge wird an der Sensoroberfläche unter Bildung von gasförmigem $\mathrm{O}_{2}$ zersetzt. Nach der Sauerstoffsättigung des Gels bildeten sich Blasen zwischen der Elektrodenoberfläche und der Gelschicht. Diese Hohlräume führten zu einer Ablösung der Gelmembran und damit zu einem abrupten Stromabfall. Dieser Signalabbruch machte eine weitere Detektion von $\mathrm{H}_{2} \mathrm{O}_{2}$ mit diesen Sensoren bisher nicht mehr möglich.
Da die mit Agarose beschichteten Sensoren bis auf die Blasenbildung vielversprechende Ergebnisse zeigten, wird bei den weiteren Untersuchungen angestrebt, die Bildung der unerwünschten Hohlräume durch konstruktive Veränderungen zu reduzieren oder den Gasaustritt aus dem Elektrolyten zu erleichtern.

\section{Literatur}

[1] GOVINDARAJ, S.; MUTHURAMAN; M.S.: Systematic Review on Sterilization Methods of Implants and Medical Devices. In: International Journal of ChemTech Research 8 (2015), S. 897-911

[2] CERNY, G.: Testing of Aseptic Machines for Efficiency of Sterilization of Packaging Materials by Means of Hydrogen Peroxide. In: Packaging Technology and Science 5 (1992), S. 77-81

[3] KARYAKIN, A.A.: Advances of Prussian blue and its analogues in (bio)sensors. In: Current Opinion in Electrochemistry 5 (2017), S. 92-98

[4] BENEDET, J.; LU, D.; CIZEK, K.; LA BELLE, J.; WANG, J.: Amperometric sensing of hydrogen peroxide vapor for security screening. In: Analytical Bioanalytical Chemistry 395 (2009), S. 371-376

[5] PAGLIARO, M.; ROSSI, M.: The Future of Glycerol: New Uses of a Versatile Raw Material. RSC Green Chemistry Book Series, 2008, S. 1-9

\section{Danksagung}

Die vorgestellten Ergebnisse sind Teil eines Projekts, finanziert vom Europäischen Fonds für Regionale Entwicklung (EFRE), FKZ 100270258. Das Kurt-Schwabe-Institut für Mess- und Sensortechnik e.V. Meinsberg wird mitfinanziert durch Steuermittel auf der Grundlage des vom Sächsischen Lamgtag beschlossenen Haushaltes. 